\title{
GAUGES AND THEIR DENSITIES
}

\author{
BY \\ GERALD FREILICH
}

1. Introduction. The method of constructing measures from gauges in a metric space is particularly important in measure theory. Examples of such include the family of Hausdorff measures in a general metric space, and the integralgeometric (Favard) measures in Euclidean space. The original idea is due to Carathéodory [2]. In generating a measure from a gauge, one considers countable coverings of a given set by elements in the domain of the gauge and only considers the sum of the corresponding values of the gauge. The main object of this paper is to investigate the relationship of the individual terms in the sum to the set that is covered. In particular, one can ask how well the gauge value of a single element of the covering approximates the measure of that part of the covered set within the given element. This leads naturally to the definition of upper and lower gauge densities to be found in $\$ 3$. In this paper, results are only obtained for upper densities.

In $\S 3$, under rather general conditions, it is proved that the upper gauge density is not less than unity for almost all points of a given set (Theorems 3.2, 3.3, 3.4). Here as in the remainder of the paper, measurability of the set is not assumed.

In $\S 4$, more conditions are imposed on both the gauge and the metric space, leading to the definition of diametric gauges, However, the concept is still general enough to include sphere and Hausdorff measure in Euclidean space or in sufficiently well-behaved manifolds. For diametric gauges and for sets of finite (outer) measure, it is proved that the upper density is unity almost everywhere in the set (Theorem 4.4). Theorem 4.2 appeared in the special case of Hausdorff measure on the real line in a paper of Besicovitch and Moran [1]. Finally, Theorem 4.5 states that for diametric gauges and sets of finite measure, one can use the gauge to approximate simultaneously in the large and in the small; thus one can cover with sets, for most of which the gauge value approximates the measure covered, and such that the sum of the gauge values approximates the measure of the whole set.

A good summary of earlier and related results can be found in Federer [3, especially §3].

2. Preliminaries. Throughout this paper, the following will be assumed:

$\varnothing$ denotes the null set.

$\infty / a=\infty$ if $a>0$.

Received by the editors June 28,1965 . 
If $(R, \rho)$ is a metric space, $x \in R, 0<r<\infty$, then $U(x, r)$ will denote $\{y \in R \mid \rho(x, y)<r\}$. Also, for $A \subset R, \operatorname{diam} A$ will denote the diameter of the set $A$.

DefinItion 2.1. Let $(R, \rho)$ be a metric space. We shall say that $g$ is a gauge over $R$ if $g$ is a nonnegative real-valued function defined over a class $M$ of subsets of $R, \varnothing \in M$ and $g(\varnothing)=0$. We shall say that the (outer) measure $m$ is generated by the gauge $g$ if for all $A \subset R$,

$$
m(A)=\lim _{r \rightarrow 0^{+}} \inf \left\{\sum_{i=1}^{\infty} g\left(M_{i}\right) \mid A \subset \bigcup_{i=1}^{\infty} M_{i}, \operatorname{diam} M_{i}<r, M_{i} \in M\right\} .
$$

As usual, a set $A \subset R$ is said to be $m$-measurable if for all $B \subset R$,

$$
m(B)=m(B \cap A)+m(B-A) .
$$

Lemma 2.2. Let $(R, \rho)$ be a metric space, $g$ be a gauge over $R$ with domain $M$, $m$ the outer measure generated by $g, A \subset R, m(A)<\infty$. Then for $\varepsilon>0$, there exists a $\delta>0$ such that

$$
\begin{array}{r}
\left(M_{0}=\bigcup_{i=1}^{\infty} M_{i}, M_{i} \in M, M_{0} \text { is } m \text {-measurable, } \operatorname{diam} M_{i}<\delta\right) \\
\Rightarrow\left(\sum_{i=1}^{\infty} g\left(M_{i}\right)>m\left(A \cap M_{0}\right)-\varepsilon\right) .
\end{array}
$$

Proof. Given $\varepsilon>0$, choose $\delta>0$ so that

$$
\left(A \subset \bigcup_{i=1}^{\infty} N_{i}, \operatorname{diam} N_{i}<\delta, N_{i} \in M\right) \Rightarrow\left(\sum_{i=1}^{\infty} g\left(N_{i}\right)>m(A)-\frac{\varepsilon}{2}\right) .
$$

Let $\quad M_{i} \in M, \quad \operatorname{diam} M_{i}<\delta, \quad M_{0}=\bigcup_{i=1}^{\infty} M_{\imath}$ be $m$-measurable. Then $m(A)=m\left(A \cap M_{0}\right)+m\left(A-M_{0}\right)$. Choose $P_{i} \in M$ so that

$$
\operatorname{diam} P_{i}<\delta, \quad A-M_{0} \subset \bigcup_{i=1}^{\infty} P_{i}, \sum_{i=1}^{\infty} g\left(P_{i}\right)<m\left(A-M_{0}\right)+\frac{\varepsilon}{2} .
$$

Then since $\left\{M_{i}\right\} \cup\left\{P_{i}\right\}$ covers $A$ and each set in the covering has diameter less than $\delta$, it follows that

$$
\begin{aligned}
\sum_{i=1}^{\infty} g\left(M_{i}\right)+\sum_{i=1}^{\infty} g\left(P_{i}\right) & >m(A)-\frac{\varepsilon}{2} \\
& =m\left(A \cap M_{0}\right)+m\left(A-M_{0}\right)-\frac{\varepsilon}{2} \\
& >m\left(A \cap M_{0}\right)+\sum_{i=1}^{\infty} g\left(P_{i}\right)-\varepsilon, \\
\sum_{i=1}^{\infty} g\left(M_{i}\right) & >m\left(A \cap M_{0}\right)-\varepsilon .
\end{aligned}
$$

REMARK. In Lemma 2.2, the condition that $M_{0}$ be $m$-measurable is usually 
superfluous in application. This is because the outer measure $m$ has the property of being additive on two sets a positive distance apart, so that Borel sets are $m$ measurable. In many applications, $M$ consists of Borel sets only.

\section{Gauge densities.}

Definition 3.1. Let $g$ be a gauge over $R$ with domain $M$, and let $m$ be the generated outer measure. For $x \in R, A \subset R$, we define the upper and lower gauge densities of $A$ at $x$ by

$$
\begin{aligned}
& D^{*} g_{A}(x)=\lim _{n \rightarrow \infty} \sup _{N \in M_{n}} \frac{m(A \cap N)}{g(N)}, \\
& D^{*} g_{A}(x)=\lim _{n \rightarrow \infty} \inf _{N \in M_{n}} \frac{m(A \cap N)}{g(N)},
\end{aligned}
$$

where $M_{n}=\{N \in M \mid x \in N$, $\operatorname{diam} N \leqq 1 / n, g(N) \neq 0\}$. If $D^{*} g_{A}(x)=D_{*} g_{A}(x)$, we define $D g_{A}(x)$ to be their common value.

TheOREM 3.2. Let $g$ be a gauge over $R$ such that every element of $M$ is $m$ measurable, $A \subset R, m(A)<\infty$. Then $D^{*} g_{A}(x) \geqq 1$ for all points of $A$ except for a set of m-measure zero.

Proof. Let $1>\varepsilon>0$ and define $\varepsilon_{n}=\varepsilon^{2} / 2^{2 n+4}$ for each positive integer $n$. By Lemma 2.2 , choose $0<\delta_{n}<1 / n$ so that if $M_{0}=\bigcup_{i=1}^{\infty} M_{i}, M_{i} \in M, \operatorname{diam} M_{i}<\delta_{n}$, then $\sum_{i=1}^{\infty} g\left(M_{i}\right)>m\left(A \cap M_{0}\right)-\varepsilon_{n}$. Next choose a sequence of sets $N_{i} \in M$ such that $A \subset \bigcup_{i=1}^{\infty} N_{i}$, diam $N_{i}<\delta_{n}$, and $\sum_{i=1}^{\infty} g\left(N_{i}\right)<m(A)+\varepsilon_{n}$.

Relabel those $N_{i}$ such that $g\left(N_{i}\right)>\left(1+\varepsilon / 2^{n}\right) m\left(A \cap N_{i}\right)>0$ by the symbols $Q_{j}$; relabel those $N_{i}$ such that $g\left(N_{i}\right)=0$ by the symbols $R_{j}$; relabel those $N_{i}$ such that $g\left(N_{i}\right)>0=m\left(A \cap N_{i}\right)$ by the symbols $T_{j}$; and relabel the remaining $N_{i}$ by the symbols $V_{j}$. For each $Q_{j}$ choose a sequence of sets $W_{j, k} \in M$ such that $A \cap Q_{j} \subset \bigcup_{k} W_{j, k}, \quad \operatorname{diam} W_{j, k}<\delta_{n}$ and $\Sigma_{k} g\left(W_{j, k}\right)<\left(1+\varepsilon / 2^{n+1}\right) \cdot m\left(A \cap Q_{j}\right)$. Since $\left\{W_{j, k}\right\} \cup\left\{R_{j}\right\} \cup\left\{T_{j}\right\} \cup\left\{V_{j}\right\}$ cover $A$ by sets of diameter less than $\delta_{n}$,

$$
\sum_{j, k} g\left(W_{j, k}\right)+\sum_{j} g\left(R_{j}\right)+\sum_{j} g\left(T_{j}\right)+\sum_{j} g\left(V_{j}\right)>m(A)-\varepsilon_{n} .
$$

Also

Hence

$$
\sum_{j} g\left(Q_{j}\right)+\sum_{j} g\left(R_{j}\right)+\sum_{j} g\left(T_{j}\right)+\sum_{j} g\left(V_{j}\right)<m(A)+\varepsilon_{n}
$$

$$
\begin{aligned}
2 \varepsilon_{n} & >\sum_{j} g\left(Q_{j}\right)-\sum_{j, k} g\left(W_{j, k}\right)=\sum_{j}\left(g\left(Q_{j}\right)-\sum_{k} g\left(W_{j, k}\right)\right) \\
& >\sum_{j}\left(1+\frac{\varepsilon}{2^{n}}-1-\frac{\varepsilon}{2^{n+1}}\right) \cdot m\left(A \cap Q_{j}\right)=\frac{\varepsilon}{2^{n+1}} \sum_{j} m\left(A \cap Q_{j}\right),
\end{aligned}
$$

so that

$$
\sum_{j} m\left(A \cap Q_{j}\right)<\frac{\varepsilon}{2^{n+2}}
$$


An application of Lemma 2.2 shows that

$$
m\left(A \cap \bigcup_{j} R_{j}\right)<\sum_{j} g\left(R_{j}\right)+\varepsilon_{n}=\varepsilon_{n}<\frac{\varepsilon}{2^{n+2}} .
$$

Also

$$
0 \leqq m\left(A \cap \bigcup_{j} T_{j}\right) \leqq \sum_{j} m\left(A \cap T_{j}\right)=0 .
$$

Hence if we define $B_{n}=\bigcup_{j} V_{j}$, then since $A-B_{n}$ is covered by the sets $\left\{Q_{j}\right\}$, $\left\{R_{j}\right\},\left\{T_{j}\right\}$, it follows that

$$
m\left(A-B_{n}\right) \leqq \sum_{j} m\left(A \cap Q_{j}\right)+m\left(A \cap \bigcup_{j} R_{j}\right)+m\left(A \cap \bigcup_{j} T_{j}\right)<\frac{\varepsilon}{2^{n+1}} .
$$

Also, if $x \in B_{n}$, then $x \in V_{j}$ for some $j$, and $g\left(V_{j}\right) \neq 0, m\left(A \cap V_{j}\right) / g\left(V_{j}\right) \geqq\left(1+\varepsilon / 2^{n}\right)^{-1}$. If we next define $B=\bigcap_{n=1}^{\infty} B_{n}$, then $m(A-B) \leqq \sum_{n=1}^{\infty} m\left(A-B_{n}\right)<\varepsilon$. It is obvious that if $x \in B$, then $D^{*} g_{A}(x) \geqq 1$. Since $\varepsilon$ was arbitrary, the proof is complete.

THEOREM 3.3. Let $g$ be a gauge over $R$ such that if $\varnothing \neq N \in M$, then $g(N)>0$. If $A \subset R, m(A)<\infty$, then $D^{*} g_{A}(x) \geqq 1$ for all points of $A$ except for a set of m-measure zero.

Proof. Let $1>\varepsilon>0$ and define $\varepsilon_{n}=\varepsilon^{2} / 2^{2 n+4}$ for each positive integer $n$. From the definition of $m(A)$, choose $0<\delta_{n}<1 / n$ so that if $M_{i} \in M, A \subset \bigcup_{i=1}^{\infty} M_{i}$, $\operatorname{diam} M_{i}<\delta_{n}$, then $\sum_{i=1}^{\infty} g\left(M_{i}\right)>m(A)-\varepsilon_{n}$. Next choose a sequence of sets $N_{i} \in M$ such that $A \subset \bigcap_{i=1}^{\infty} N_{i}$, $\operatorname{diam} N_{i}<\delta_{n}$ and $\sum_{i=1}^{\infty} g\left(N_{i}\right)<m(A)+\varepsilon_{n}$. The proof of Theorem 3.2 now carries over if one observes that the class $\left\{R_{j}\right\}$ is either empty or consists of $\varnothing$ alone and hence no application of Lemma 2.2 is required

THEOREM 3.4. Let

(1) $g$ be a gauge over $R$,

(2) $A \subset R$ be arbitrary,

(3) $R$ be separable,

(4) there exist a $\delta>0$ such that if $x \in R, 0<r<\delta$, then $U(x, r) \in M$ and $g(U(x, r))>0$,

(5) every element of $M$ be m-measurable.

Then $D^{*} g_{A}(x) \geqq 1$ for all points of $A$ except for a set of $m$-measure zero.

Proof. We first note that because of condition (5), every set is contained in an $m$-measurable set of the same measure. Hence for any sequence of sets $A_{i}$, $m\left(\bigcup_{i=1}^{\infty} A_{i}\right)=\lim _{n \rightarrow \infty} m\left(\bigcup_{i=1}^{n} A_{i}\right)$.

Let $Y$ be a countable set of points dense in $R$. Let $B=\left\{x \in A \mid D^{*} g_{A}(x)<1\right\}$ and assume $m(B)>0$. For each $x \in B$, choose a sphere $U_{x}=U(y, r) \in M$ with $y \in Y, x \in U_{x}, r$ a positive rational number, and $m\left(U_{x} \cap A\right)<g\left(U_{x}\right)$. Since the collection $\left\{U_{x} \mid x \in B\right\}$ is countable, order them as $U_{1}, U_{2}, \cdots$. Choose the integer 
$n$ so that $m\left(B \cap \bigcup_{i=1}^{n} U_{i}\right)>0$. Let $A_{1}=A \cap \bigcup_{i=1}^{n} U_{i}, B_{1}=B \cap \bigcup_{i=1}^{n} U_{i}$. Then $B_{1} \subset A_{1}, m\left(B_{1}\right)>0, m\left(A_{1}\right)<\infty$ and for $x \in B_{1}, D^{*} g_{A}(x) \leqq D^{*} g_{A 1}(x)<1$. Since Theorem 3.2 is contradicted, the proof is complete.

REMARK. In Theorems 3.2 and 3.4, the condition that every element of $M$ be $m$-measurable is usually satisfied in applications, as has been already remarked. We now give an example of a gauge $g$ such that no nonnull element of $M$ is $m$ measurable. If $M_{1}$ is the set of open intervals in Euclidean 1-space $E^{\mathbf{1}}$, and $g_{1}(I)=\operatorname{diam} I$ for an open interval $I$, then the generated measure is Lebesgue 1-dimensional measure, $L_{1}$. Let $M$ be the set of all subsets of $E^{1}$ of the form $\varnothing$ or $(a, b) \cup C$, where $C$ is $L_{1}$ nonmeasurable, $C \subset\left(b, b+(b-a)^{2}\right), a<b$. Again let $g(N)=\operatorname{diam} N$ for $N \in M$. Then $g$ generates $L_{1}$ and no nonnull member of $M$ is $L_{1}$-measurable.

4. Diametric gauges. We now fix the metric space $(R, \rho)$ and the gauge $g$. Throughout the remainder of this paper, we shall make certain assumptions on the gauge and the metric space which we formalize in the following definition.

Definition 4.1. $g$ is a diametric gauge over $R$ with domain $M$ if

(i) $g$ is a gauge over $R$ with $m$ the generated outer measure,

(ii) there exists a $\delta>0$ such that if $x \in R, 0<r \leqq \delta$, then $U(x, r) \in M$ and $\operatorname{diam} U(x, r)>r$.

(iii) every member of $M$ is $m$-measurable,

(iv) there exist nondecreasing functions $f_{1}$ and $f_{2}$ on the nonnegative real numbers and a positive constant $k$ such that $f_{1}(2 r) \leqq k \cdot f_{2}(r)$ and if $N \in M$, $\operatorname{diam} N=r$, then $f_{2}(r) \leqq g(N) \leqq f_{1}(r)$,

(v) there exists a positive integer $L$ such that any open sphere of radius $6 r$ can be covered by $L$ open spheres of radius $r$ ( $L$ independent of $r$ ).

REMARK. In Definition 4.1 (iv) one can replace $2 \cdot r$ by $p \cdot r$ where $p$ is any fixed number greater than one, and similarly in $4.1(\mathrm{v})$ the radius $6 \cdot r$ can be replaced by $p \cdot r$. It is obvious that the definition is then unchanged. In what follows, condition $4.1(\mathrm{v})$ can be weakened so that it holds only for $r$ sufficiently small.

THEOREM 4.2. Let $g$ be a diametric gauge over $R, A \subset R, m(A)<\infty$. For every $p>1, \varepsilon>0$, there exists $a \delta>0$ and sets $C$ and $B=A-C$ such that:

(i) $C$ is the union of elements of $M$,

(ii) $m(A \cap C) \leqq \varepsilon, m(B) \geqq m(A)-\varepsilon$,

(iii) if $x \notin C, x \in N \in M$, $\operatorname{diam} M \leqq \delta$, then $m(A \cap N) \leqq p \cdot g(N)$,

(iv) for all $N \in M$ with $\operatorname{diam} N \leqq \delta, m(B \cap N) \leqq p \cdot g(N)$.

Proof. We exclude the trivial case $m(A)=0$. Let $L$ be an integer such that for all $0<r<\infty$, an arbitrary sphere in $R$ of radius $6 r$ can be covered by $L$ open spheres of radius $r$. Choose a constant $k>1$ such that $f_{1}(2 r) \leqq k \cdot f_{2}(r)$, where $f_{1}$ and $f_{2}$ are the functions of 4.1 (iv). 
Let $u=\varepsilon(p-1)(p k L)^{-1}$, and apply Lemma 2.2 to choose $\delta>0$ so that $U(x, r) \in M, \operatorname{diam} U(x, r)>r$ for $x \in R, 0<r \leqq \delta$, and

$$
\left(M_{0}=\bigcup_{i=1}^{\infty} M_{i}, M_{i} \in M, \operatorname{diam} M_{i} \leqq \delta\right) \Rightarrow\left(\sum_{i=1}^{\infty} g\left(M_{i}\right)>m\left(A \cap M_{0}\right)-u\right) .
$$

Next, define $C=\{x \in R \mid$ for some $N \in M, x \in N, \operatorname{diam} N \leqq \delta, \quad m(A \cap N)$ $\geqq p \cdot g(N)\}$, and for each nonnegative integer $t$, define

$$
\begin{aligned}
D_{t} & =\left\{N \in M \mid \frac{\delta}{2^{t+1}}<\operatorname{diam} N \leqq \frac{\delta}{2^{t}}, m(A \cap N) \geqq p \cdot g(N)\right\}, \\
D & =\bigcup_{t=0}^{\infty} D_{t},
\end{aligned}
$$

so that $C=\bigcup_{N \in D} N$.

For each $N \in D_{t}$, we associate a sphere $V(N)$ by choosing $x \in N$ and taking $V(N)=U\left(x, 3 \delta / 2^{t}\right)$.

If $D_{0} \neq \varnothing$, choose $N_{0,1} \in D_{0}$ so that

$$
m\left(A \cap N_{0,1}\right)>\frac{1}{k} \sup _{N \in D_{0}} m(A \cap N) .
$$

Then $V\left(N_{0,1}\right)$ can be covered by $L$ open spheres of radius $\delta / 2$; call them $V_{1}, \cdots, V_{L}$. If $V_{i} \in D_{0}$, then $m\left(A \cap V_{i}\right)<k \cdot m\left(A \cap N_{0,1}\right)$. If $V_{i} \notin D_{0}$, then since $\operatorname{diam} V_{i}<2 \cdot \operatorname{diam} N_{0,1}$, it follows that $m\left(A \cap V_{i}\right)<p \cdot g\left(V_{i}\right) \leqq p \cdot k g\left(N_{0,1}\right)$ $\leqq k \cdot m\left(A \cap N_{0,1}\right)$. Hence

$$
m\left(A \cap V\left(N_{0,1}\right)\right) \leqq \sum_{i=1}^{L} m\left(A \cap V_{i}\right) \leqq k \cdot L \cdot m\left(A \cap N_{0,1}\right) .
$$

Let $D_{0,1}=\left\{N \in D_{0} \mid N \cap N_{0,1}=\varnothing\right\}$. If $D_{0,1}=\varnothing$, the procedure stops. If $D_{0,1} \neq \varnothing$, choose $N_{0,2} \in D_{0,1}$ so that $m\left(A \cap N_{0,2}\right)>(1 / k) \sup _{N \in D_{0,1}} m(A \cap N)$. Then $V\left(N_{0,2}\right)$ can be covered by $L$ open spheres of radius $\delta / 2$; call them $W_{1}, \cdots, W_{L}$.

If $W_{i} \cap N_{0,1} \neq \varnothing$, then $W_{i} \subset V\left(N_{0,1}\right)$. If $W_{i} \cap N_{0,1}=\varnothing$ and $W_{i} \notin D_{0}$, then

$$
m\left(A \cap W_{i}\right)<p \cdot g\left(W_{i}\right) \leqq p \cdot k \cdot g\left(N_{0,2}\right) \leqq k \cdot m\left(A \cap N_{0,2}\right) .
$$

If $W_{i} \in D_{0,1}$, then $m\left(A \cap W_{i}\right)<k \cdot m\left(A \cap N_{0,2}\right)$. Hence

so that

$$
\left.m\left(A \cap\left(V\left(N_{0,2}\right)-V\left(N_{0,1}\right)\right)\right) \leqq \sum_{W_{i}, N_{01}=\varnothing} m\left(A \cap W_{i}\right)\right) \leqq k L m\left(A \cap N_{0}{ }_{2}\right),
$$

$$
\begin{aligned}
m\left(A \cap\left(V\left(N_{0,1}\right) \cup V\left(N_{0,2}\right)\right)\right) \leqq & m\left(A \cap V\left(N_{0,1}\right)\right) \\
& +m\left(A \cap\left(V\left(N_{0,2}\right)-V\left(N_{0,1}\right)\right)\right) \\
\leqq & k \cdot L\left(m\left(A \cap N_{0,1}\right)+m\left(A \cap N_{0,2}\right)\right) \\
= & k \cdot L \cdot m\left(A \cap\left(N_{0,1} \cup N_{0,2}\right)\right) .
\end{aligned}
$$


Since $m(A)<\infty$, the above procedure can be continued only a finite number of times, say $q(0)$ (with $q(0)$ possibly 0 ), to obtain $N_{0,1}, N_{0,2}, \cdots, N_{0, q(0)}$ with the properties:

(i) $N_{0, i} \in D_{0}$ for $1 \leqq i \leqq q(0)$,

(ii) $N_{0, i} \cap N_{0, j}=\varnothing$ for $i \neq j$,

(iii) $m\left(A \cap \bigcup_{i=1}^{q(0)} V\left(N_{0, i}\right)\right) \leqq k \cdot L \cdot m\left(A \cap \bigcup_{i=1}^{q(0)} N_{0, i}\right)$,

(iv) $\bigcup_{N \in D_{0}} N \subset \bigcup_{i=1}^{q(0)} V\left(N_{0, i}\right)$.

The last condition is explained by the fact that when the procedure cannot be continued, an arbitrary $N \in D_{0}$ must intersect some $N_{0, i}$ and then $N \subset V\left(N_{0, i}\right)$.

Next, let

$$
D_{1,0}=\left\{N \in D_{1} \mid N \cap \bigcup_{i=1}^{q(0)} N_{0, i}=\varnothing\right\}
$$

We shall only consider the nontrivial case when $D_{1,0} \neq \varnothing$. Choose $N_{1,1} \in D_{1,0}$ so that $m\left(A \cap N_{1,1}\right)>(1 / k) \sup _{N \in D 1,0} m(A \cap N)$. Then $V\left(N_{1,1}\right)$ can be covered by $L$ spheres of radius $\delta / 4$, to be denoted by $Z_{1}, Z_{2}, \cdots, Z_{L}$. If $Z_{i} \cap \bigcup_{i=1}^{q(0)} N_{0, i} \neq \varnothing$, then $Z_{i} \subset \bigcup_{i=1}^{q(0)} V\left(N_{0, i}\right)$. If $Z_{i} \cap \bigcup_{i=1}^{q(0)} N_{0, i}=\varnothing, Z_{i} \notin D_{1,0}$, then $Z_{i} \notin D_{1}$, so that $m\left(A \cap Z_{i}\right)<p \cdot g\left(Z_{i}\right) \leqq p \cdot k \cdot g\left(N_{1,1}\right) \leqq k m\left(A \cap N_{1,1}\right)$. If $Z_{i} \in D_{1,0}$, then $m\left(A \cap Z_{i}\right)<. k m\left(A \cap N_{1,1}\right)$. Hence

$$
\begin{aligned}
& m\left(A \cap\left(V\left(N_{1,1}\right)-\bigcup_{i=1}^{q(0)} V\left(N_{0, i}\right)\right)\right) \leqq k \operatorname{Lm}\left(A \cap N_{1,1}\right), \\
& m\left(A \cap\left(V\left(N_{1,1}\right) \cup \bigcup_{i=1}^{q(0)} V\left(N_{0, i}\right)\right)\right) \leqq k \operatorname{Lm}\left(A \cap\left(N_{1,1} \cup \bigcup_{i=1}^{q(0)} N_{0, i}\right)\right) .
\end{aligned}
$$

The above procedure can be applied only a finite number of times to the class $D_{1}$, and a similar procedure can be carried on to all $D_{i}$ to finally obtain a collection of sets $N_{i, j}(i \geqq 0, i \leqq j \leqq q(i))$ with the properties:

(i) $N_{i, j} \in D_{i}$ for $i \geqq 0,1 \leqq j \leqq q(i)$,

(ii) $N_{i, j} \cap N_{r, s}=\varnothing$ if either $i \neq r$ or $j \neq s$,

(iii) $m\left(A \cap \bigcup_{i=0}^{\infty} \bigcup_{j=1}^{q(i)} V\left(N_{i, j}\right)\right) \leqq k L \cdot m\left(A \cap \bigcup_{=0}^{\infty} \bigcup_{j=1}^{q(i)} N_{i, j}\right)$,

(iv) $C=\bigcup_{N \in D} N \subset \bigcup_{i=0}^{\infty} \bigcup_{j=1}^{j} V\left(N_{i, j}\right)$.

By the choice of $\delta$ and the fact that $\operatorname{diam} N_{i, j} \leqq \delta$ for all $i$ and $j$, it follows that

$$
\begin{aligned}
\sum_{i=0}^{\infty} \sum_{j=1}^{q(i)} m\left(A \cap N_{i, j}\right) & =m\left(A \cap \bigcup_{i=0}^{\infty} \bigcup_{j=1}^{q(i)} N_{i, j}\right)<\sum_{i=0}^{\infty} \sum_{j=1}^{q(i)} g\left(N_{i, j}\right)+u \\
& \leqq \frac{1}{p} \sum_{i=0}^{\infty} \sum_{j=1}^{q(i)} m\left(A \cap N_{i, j}\right)+u,
\end{aligned}
$$

so that

Hence

$$
\sum_{i=0}^{\infty} \sum_{j=1}^{q(i)} m\left(A \cap N_{i, j}\right) \leqq \frac{p u}{p-1}
$$




$$
\begin{aligned}
m(A \cap C) & \leqq m\left(A \cap \bigcup_{i=0}^{\infty} \bigcup_{j=1}^{q(i)} V\left(N_{i, j}\right)\right. \\
& \leqq k \cdot L \cdot m\left(A \cap \bigcup_{i=0}^{\infty} \bigcup_{j=1}^{q(i)} N_{i, j}\right) \\
& \leqq k \cdot L \cdot \sum_{i=0}^{\infty} \sum_{j=1}^{q(i)} m\left(A \cap N_{i, j}\right) \\
& \leqq \frac{p \cdot k \cdot L \cdot u}{p-1}=\varepsilon .
\end{aligned}
$$

Let $B=A-C$. Then

$$
m(A) \leqq m(A-C)+m(A \cap C) \leqq m(B)+\varepsilon .
$$

Also, from the definition of $C$, it follows that if $x \notin C, x \in N \in M$, diam $N \leqq \delta$, then $m(A \cap N)<p \cdot g(N)$. Finally, if $N \in M$, diam $N \leqq \delta$ and $m(B \cap N)>p \cdot g(N)$, then $m(A \cap N)>p \cdot g(N)$. Hence $N \in D$ and therefore $N \subset C, N \cap B=\varnothing$, contradicting $m(N \cap B)>0$. The proof is complete.

Corollary 4.3. Let $g$ be a diametric gauge over $R, A \subset R, m(A)<\infty$. For $\varepsilon>0$ there exist sets $C$ and $B=A-C$ such that

(i) $C$ is the union of elements of $M$,

(ii) $m(A \cap C) \leqq \varepsilon, m(B) \geqq m(A)-\varepsilon$,

(iii) for each $p>1$ there is a $\delta>0$ such that if $x \notin C, x \in N \in M$, $\operatorname{diam} N \leqq \delta$, then $m(A \cap N) \leqq p \cdot g(N)$,

(iv) for each $p>1$, there is a $\delta>0$ such that $m(B \cap N) \leqq p \cdot g(N)$ for all $N \in M$ with $\operatorname{diam} N \leqq \delta$.

Proof. For $p_{j}=1+1 / j$, use Theorem 4.2 to choose $C_{j}$, a union of elements of $M$, and $\delta_{j}>0$ such that:

(i) $m\left(A \cap C_{j}\right) \leqq \varepsilon / 2^{j}$,

(ii) if $x \notin C_{j}, x \in N \in M$, $\operatorname{diam} N \leqq \delta_{j}$, then $m(A \cap N) \leqq p_{j} \cdot g(N)$,

(iii) $m\left(\left(A-C_{j}\right) \cap N\right) \leqq p_{j} \cdot g(N)$ for all $N \in M$ with $\operatorname{diam} N \leqq \delta_{j}$.

Define $C=\bigcup_{j=1}^{\infty} C_{j}$ and $B=A-C$. The remaining details of the proof are easily checked.

REMARK. In a sense, Theorem 4.2 and Corollary 4.3 represent the best possible result. For let $R$ be the Euclidean plane, $M$ the set of all subsets of the plane, $g(N)=\operatorname{diam} N$ for $N \in M$, so that $g$ generates Hausdorff linear measure. Let

$$
\begin{gathered}
A=\left\{(x, y) \mid x^{2}+y^{2}=1\right\} \cup\{(x, y) \mid x=0,-1 \leqq y \leqq 1\} \\
\cup\{(x, y) \mid y=0,-1 \leqq x \leqq 1\} .
\end{gathered}
$$

Then for the conclusions of either the theorem or corollary to hold, a set of positive measure must be taken away from $A$. 
THEOREM 4.4. Let $g$ be a diametric gauge over $R, A \subset R, m(A)<\infty$.

Then $D^{*} g_{A}(x)=1$ for all points of $A$ except for a set of $m$-measure zero.

Proof. Apply Theorem 3.2 and Corollary 4.3.

THEOREM 4.5. Let $g$ be a diametric gauge over $R, A \subset R, m(A)<\infty$. For each $\varepsilon>0, p>1$, there exist two sequences of sets $\left\{N_{i}\right\} \subset M,\left\{Q_{i}\right\} \subset M$ such that:

(i) $A \subset \bigcup_{i} N_{i} \cup \bigcup_{i} Q_{i}$,

(ii) $\operatorname{diam} N_{i} \leqq \varepsilon, \operatorname{diam} Q_{i} \leqq \varepsilon$ for each $i$,

(iii) $m(A)-\varepsilon \leqq \Sigma_{i} g\left(N_{i}\right)+\Sigma_{i} g\left(Q_{i}\right) \leqq m(A)+\varepsilon$,

(iv) $\Sigma_{i} g\left(Q_{i}\right) \leqq \varepsilon$,

(v) $p^{-1} \cdot g\left(N_{i}\right) \leqq m\left(A \cap N_{i}\right) \leqq p g\left(N_{i}\right)$ for each $i$.

Proof. We exclude the trivial case $m(A)=0$. Without loss of generality, we assume $(p-1) \varepsilon<m(A)$ and $p<2$. Apply Theorem 4.2 and Lemma 2.2 to choose a set $C$ and a $\delta, 0<\delta<\varepsilon$, such that:

(i) $\left(M_{i} \in M, \operatorname{diam} M_{i} \leqq \delta\right) \Rightarrow\left(\sum_{i=1}^{\infty} g\left(M_{i}\right) \geqq m\left(A \cap \bigcup_{i=1}^{\infty} M_{i}\right)-\varepsilon / 4\right)$,

(ii) $m(A \cap C)<\varepsilon / 4$,

(iii) $(x \notin C, x \in N \in M, \operatorname{diam} N \leqq \delta) \Rightarrow\left\{\begin{array}{l}m(A \cap N) \leqq\left(1-\frac{(p-1) \varepsilon}{16 m(A)}\right)^{-1} \cdot g(N), \\ m(A \cap N) \leqq p \cdot g(N) .\end{array}\right.$

Let $B=A-C$. Choose a sequence $\left\{T_{i}\right\}$ such that each $T_{i} \in M$, diam $T_{i} \leqq \delta$, $T_{i} \cap B \neq \varnothing, \quad B \subset \bigcup_{i} T_{i}, \quad \Sigma_{i} g\left(T_{i}\right) \leqq m(B)+(p-1) \varepsilon / 16$. Denote by $\left\{V_{i}\right\}$ those $T_{i}$ for which $g\left(T_{i}\right)>p \cdot m\left(B \cap T_{i}\right)$ and denote the remaining $T_{i}$ by $\left\{N_{i}\right\}$. Then. $q=(1-(p-1) \varepsilon / 16 m(A))^{-1}$,

$$
\begin{aligned}
m(B)+\frac{(p-1) \varepsilon}{16} & \geqq \sum_{i} g\left(T_{i}\right)=\sum_{i} g\left(V_{i}\right)+\sum_{i} g\left(N_{i}\right) \\
& \geqq p \sum_{i} m\left(B \cap V_{i}\right)+\frac{1}{q} \sum_{i} m\left(A \cap N_{i}\right) \\
& \geqq(p-1) \sum_{i} m\left(B \cap V_{i}\right)+\frac{1}{q} m(B),
\end{aligned}
$$

so that

$$
\begin{aligned}
\sum_{i} m\left(B \cap V_{i}\right) & \leqq\left(\left(1-\frac{1}{q}\right) m(B)+\frac{(p-1) \varepsilon}{16}\right)(p-1)^{-1} \leqq \frac{\varepsilon}{8}, \\
m\left(B \cap \bigcup_{i} V_{i}\right) & \leqq \frac{\varepsilon}{8}
\end{aligned}
$$

Hence 


$$
\begin{aligned}
& \sum_{i} g\left(N_{i}\right) \geqq m\left(A \cap \bigcup_{i} N_{i}\right)-\frac{\varepsilon}{4} \geqq m\left(B-\bigcup_{i} V_{i}\right)-\frac{\varepsilon}{4} \\
& \geqq m(B)-m\left(B \cap \bigcup_{i} V_{i}\right)-\frac{\varepsilon}{4} \geqq m(B)-\frac{3 \varepsilon}{8} \\
& \sum g\left(V_{i}\right) l=\sum_{i} g\left(T_{i}\right)-\sum_{i} g\left(N_{i}\right) \leqq m(B) \\
&+\frac{\varepsilon}{16}-\left(m(B)-\frac{3 \varepsilon}{8}\right)<\frac{\varepsilon}{2} .
\end{aligned}
$$

Finally, choose a sequence $\left\{R_{i}\right\}$ such that for each $i, R_{i} \in M$, diam $R_{i} \leqq \delta$, $A \cap C \subset \bigcup_{i} R_{i}, \Sigma_{i} g\left(R_{i}\right) \leqq \varepsilon / 2$. The proof is completed by denoting $\left\{R_{i}\right\} \cup\left\{V_{i}\right\}$ by $\left\{Q_{i}\right\}$.

\section{BIBLIOGRAPHY}

1. A. S. Besicovitch and P. A.P. Moran, The measure of product and cylinder sets, J. London Math. Soc. 20 (1945), 110-120.

2. C. Carathéodory, Uber das lineare Mass von Punktmengen-eine Verallgemeinerung des Längenbegriffs, Nachr. Ges. Wiss. Göttingen (1914), 404-426.

3. H. Federer, The $(\phi, k)$ rectifiable subsets of $n$ space, Trans. Amer. Math. Soc. 62 (1947), 114-192.

THE.CITY UNIVERSTY OF NeW YoRK, New YoRK, New YoRK 